\title{
Digital Hologram Construction and Reconstruction Optimization for Sprays using Optical Software
}

\author{
Jose Ulises Torres ${ }^{1}$, Derek Dunn-Rankin², Yu-Chien Chien ${ }^{* 2}$ \\ ${ }^{1}$ Physics Department, University of California, Irvine, Irvine, CA, USA \\ ${ }^{2}$ Mechanical and Aerospace Engineering, University of California, Irvine, Irvine, CA, USA \\ *Corresponding author email: chieny@uci.edu
}

\begin{abstract}
The goal of this research is to study the potential of simulating the digital hologram of a diesel spray and then reconstructing the spray from the hologram using the commercial optical software, Zemax ${ }^{\mathrm{TM}}$. If successful, this approach would permit design optimization of the holographic system. The limitation of a digital holography system is always depending on the capability of the equipment, such as the sensor size and resolution, the laser power, and the optical train layout, which can depend heavily on the available space. The approach in the current work is to take a recorded hologram and place it in an optical path with any attendant optical aberrations. The hologram illuminated allows its unique diffraction pattern to pass through various optical devices, and through a volume representing a highly scattering media. Preliminary simulations take an idealized USAF resolution chart hologram through an optical train in Zemax ${ }^{\mathrm{TM}}$ and digitally reconstruct the object to demonstrate the feasibility of this technique. The initial results are promising, and the reconstructions correspond well with past experimental findings, showing a lateral resolution of $24 \mu \mathrm{m}$ (element 3 in Group 4 of the USAF resolution chart) in a scattering environment with an optical depth of 7.
\end{abstract}

\section{Keywords}

Digital holography, Zemax ${ }^{\mathrm{TM}}$, Fourier Optics and signal processing, Diffraction pattern

\section{Introduction}

Holography, as it was invented, is more precisely categorized as analog in-axis holography, but this original format can suffer from poor image quality, and cumbersome physical reconstruction requirements. Recent advances in digital imaging have generated a resurgence in holographic application, including in environments that require holography to image dynamic phenomena inside high optical noise-causing environments. Hence, the current work discusses the predicted potential of digital off-axis holography to mitigate noise contributions and optical aberrations [1].

For the off-axis system, as discussed in detail by Hariharan [2], the reconstructed complex light field on the film plane is given by

$E_{R} h(x, y)=\beta \tau\left(a_{R}^{2}+a_{0}^{2}\right) E_{R}(x, y)+\beta \tau a_{R}^{2} E_{0}(x, y)+\beta \tau E_{R}^{2}(x, y) E_{0}^{*}(x, y)$

where $\mathrm{h}$ is the hologram interference pattern, and $E_{R}$ and $E_{O}$ are the complex amplitudes of the reference and object beam respectively, $a_{R}$ and $a_{0}$ are the real amplitudes, and $\beta$ and $T$ are constants. The three distinct terms in equation 1 have physical significance. The first term is the DC term and represents the undiffracted, transmitted light from the reference beam. The second term, or cross-correlation term, represents the virtual image of interest. The last term is the conjugate term and represents the real image. In off-axis reconstructions, not only are these terms separated in physical space, but also in frequency space by a Fourier transform.

The primary variable of interest for determining the quality of a holographic system is the spatial resolution $\mathrm{R}$ of the reconstructed image. The theoretical lower limit in digital holography 
is equal to the pixel spacing $\Delta x_{C C D}$. This limit is seldom reached in optically harsh conditions such as those examined in this paper because they introduce noise that degrades the virtual image quality. The level of degradation as determined from the contribution of scattered light correlates with the optical depth (OD) parameter defined as

$O D=-\ln \frac{I_{t}}{I_{i}}$

where $I_{t}$ is the intensity of the laser beam transmitted after traversing the scattering environment, and $l_{i}$ is the unaltered incident intensity. The light scattering particle size and number density, $\rho_{\mathrm{N}}$, changes the optical depth of an environment, and any scattered photons reaching the image detector are a source of noise. Fortunately, a proven technique to reduce noise contributions is coherence filtering [4]. Coherence filtering (like time gating) uses the fact that scattered photons travel a longer path length than the unscattered ballistic photons, and the path length correlates with the level of scattering. By using a laser with a very short coherence length $L$ such as a Ti:Sapphire femtosecond laser ( $L$ approximately 30 microns), only photons with a path length difference below $L$ will contribute to the hologram interference pattern. Hence, the hologram will naturally reject most of the noise from scattered photons.

While the most common measure of optical scattering harshness, the OD alone is not sufficient to fully characterize the level of scattering noise because the size of the scattering particle impacts how drastically the photon paths are disrupted. For example, a scattering environment with larger particles at lower number density can have the same OD as an environment with smaller particles at higher number density (i.e., the transmission of undeviated light is the same in both cases). However the smaller particles will scatter light to a much larger solid angle which affects the noise in the hologram and the image reconstruction. Analysing the precise relationship between these parameters can be difficult in an experimental setting due to inherent size distributions of scatterers and challenges of maintaining uniform particle mixtures.

This paper instead analyses this relationship by simplifying the problem in simulations and subsequently increases the complexity to match experimental conditions. Specifically, we begin by simplifying the distribution to be a single size of scatterer and we vary the radius and number density to achieve desired optical depth values. The next phase involves introducing optical components to magnify the hologram in order to determine the effects of additional potential aberration elements on resolution. The work is motivated by laboratory experiments that have determined that a small magnification factor does improve resolution [3], but the optimization of the achromats achieving this is yet to be optimized. The approach introduced in this paper (using commercial optical software) helps design the optical layout of a holographic system with the supposition that an optically scattering media further disrupts the collected light signals.

\section{Material and Methods}

The figure of merit or performance for the digital hologram is its spatial resolution, R. It is related to the much more commonly reported frequency resolution, $f$, by an inverselyproportional relationship $R \propto 1 / f$. Although the theoretical resolution limit is the diffractionlimit, most holographic systems are instead limited by the pixel size of the detector, as this is the smallest resolvable size. Hence, much of the effort goes into optimizing the optics and the hologram reconstruction algorithm [2]. 
The hologram recording and reconstruction process is accomplished with two pieces of software: SimuHolo, which is a MATLAB script, and Zemax OpticStudio. OpticStudio was primarily used to run ray traces in order to simulate the noise from scattering conditions as well as aberrations from lenses. The SimuHolo code was based on an off-axis holography simulation code from [2] that was modified for noise reduction by introducing a Fourier domain cropping technique. The experimental setup that is the basis for the simulations discussed here is from work at the Lasers, Flames, and Aerosols lab at UC Irvine, and is as shown in Fig. 1 [3-5]. Using SimuHolo the mirrors, beam splitters, and polarizers for the reference

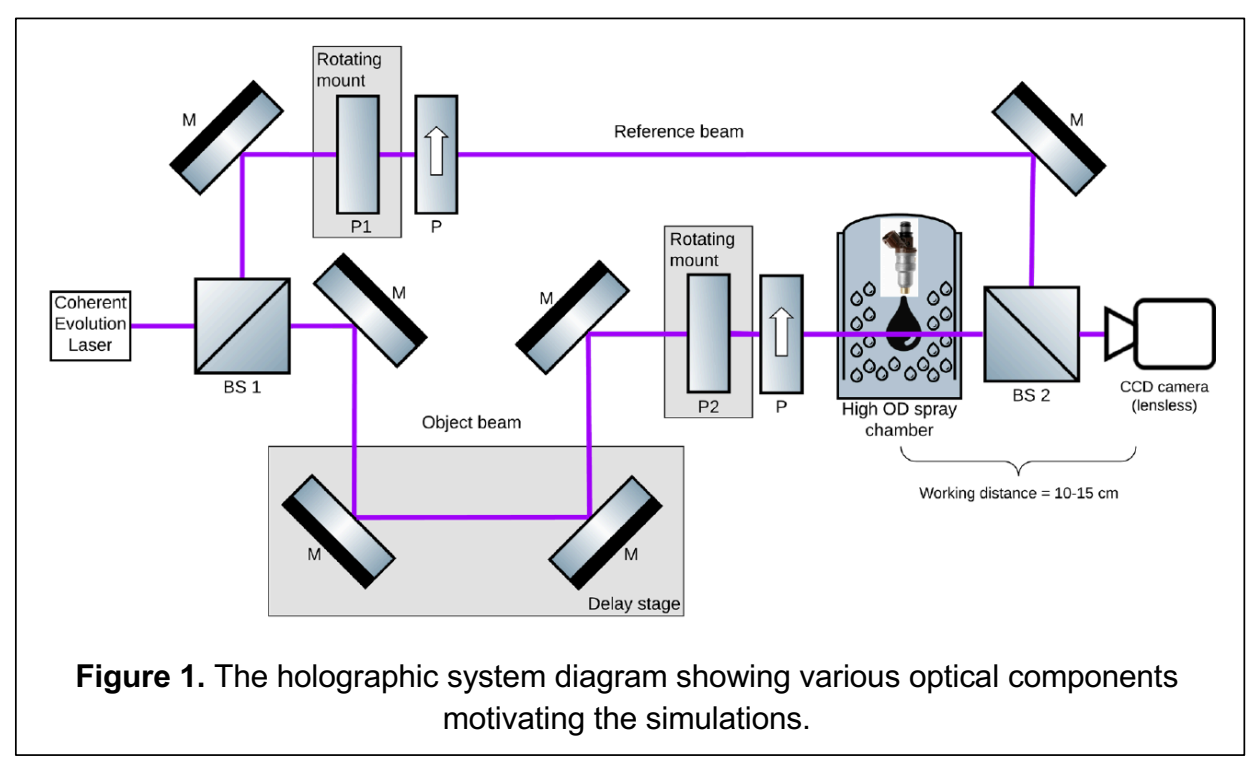

beam can be ignored, because SimuHolo does not propagate that beam, but rather sums its complex amplitude to the object beam numerically while keeping track of the phase and thus creating the interference pattern. The working distance, off-axis angle, laser wavelength, and the object are user inputs in SimuHolo. The laser coherence length, the optical components, and the scattering conditions are considered within the OpticStudio environment. The simulation process is to first use SimuHolo to record an aberration-free hologram, then to insert the hologram into the Zemax environment to simulate noise and aberrations, and finally to use SimuHolo again to perform a numerical reconstruction[6].

The algorithm behind SimuHolo uses the Angular Spectrum Method (ASM) to propagate the object beam during recording, and the Fresnel Diffraction Method (FDM) to back-propagate during reconstruction. To avoid aliasing, the critical distance and angle are calculated by the equations

$$
\begin{aligned}
& Z_{\text {min }}=\frac{4 \Delta x_{C C D}\left(D+M \Delta x_{C C D}\right)}{\lambda} \\
& \theta_{C}=\sin ^{-1} \frac{3 \lambda}{8 \Delta x_{C C D}}
\end{aligned}
$$

respectively, where $D$ is the size of the object or the largest feature in a non-continuous object. The zero-padding technique is used to increase the number of samples and therefore improve resolution. The benefit of zero padding can be quantified by considering the diffraction limits and alias-free conditions in the expression

$R=\frac{\lambda Z_{\min }}{M \Delta x_{C C D}}$

where $M$ is the number of pixels assuming a square CCD. If there are more pixels, then there are more data points, or samples. Zero-padding introduces more pixels with values of zero to the recorded hologram to accomplish this. 
SimuHolo consists of three distinct steps: propagation simulation, beam interference at detector, and reconstruction. After the first two steps, the recorded hologram is brought into the Zemax workspace where scattering noise is simulated. The hologram is then brought back to SimuHolo to be reconstructed. The break in using SimuHolo where Zemax is used occurs between the $4^{\text {th }}$ and $5^{\text {th }}$ box in the flowchart in Fig. 2 .

The object input by the user is an image in BMP file format with pixel dimensions of $M \times M$, represented by an array of those dimensions. The cropping of the frequency domain Fourier transform image around the cross-correlation term reduces the effects of noise from the DC and conjugate terms. These other terms, if not cropped out, can cover the virtual image with scattered light noise on reconstruction. We effectively isolate the cross-correlation term (corresponding to the virtual image) by selecting the region around the term from a pixel data array in MATLAB, and then proceeding with the reconstruction of this subimage. This userdefined region's size varies as the noise does, but is on the order of a tenth the size of the original input image in this context, and so is represented by an image of dimensions $\mathrm{aM} \times \mathrm{bM}$ as shown in Figure 2 where the constants a and $b$ have a value less than 1 .

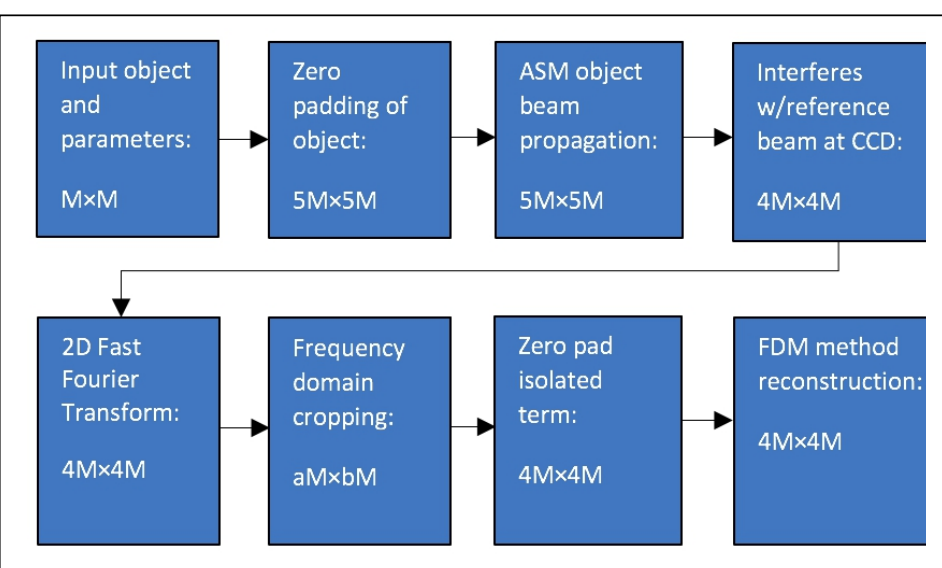

Figure 2. Flowchart of SimuHolo algorithm. $M$ is the number of pixels. The constants a and b represent values less than 1.

Zemax OpticStudio is used entirely in the Non-Sequential mode to include the scattering feature, coherence length modeling, and detector customization. The "Rectangular Volume" object within OpticStudio is used to simulate the scattering cell. Under the properties of this object is the "Volume Physics" tab in which the scatterers' radius, number density, particle index of refraction, and transmission are specified. In addition is the option to choose the scattering theory, and all simulations reported in this paper use Mie theory as is appropriate for the particle size factor $x$ regime used in the simulations. The dimensionless particle size factor is defined as $x=\frac{2 \pi r}{\lambda}$. where $r$ is the scattering particle radius and $\lambda$ is the wavelength. We varied the particle radius from a lower limit of $1 \mu \mathrm{m}$ to an upper limit of $15 \mu \mathrm{m}$. For the 400 $\mathrm{nm}$ light wavelength of the setup this range corresponds to an $\mathrm{x}$ varying from 6 to 235 , a range which justifies the use of Mie theory. The particle index of refraction and transmission factor are generally held constant for simulation studies. If we consider the particles to be completely transparent, then the transmitted intensity is nearly equal to the incident intensity and thus the transmission factor can be left at 1 . The mean path parameter in the "Volume Physics" tab is overridden if a value for number density is given, and since it is varied along with particle radius, OpticStudio internally calculates the true mean path for the ray trace.

\section{Results and Discussion}

The first task is to create a "perfect" hologram using SimuHolo, and to use this ideal hologram as a basis for all subsequent performance evaluations. A USAF Resolution chart was selected as the object, and its image was inserted into SimuHolo in the format of a $780 \times 780$ pixel BMP 
file. A wavelength of $400 \mathrm{~nm}$, and a detector pixel pitch of $6.45 \mu \mathrm{m}$ were used, because they are the values of the equipment used in the associated LFA experiments. These two variables are sufficient to calculate the critical distance and angles previously described. The $D$ variable in the critical distance equation was identified as the largest feature in the USAF chart, which is the $125 \mu \mathrm{m}$ width of the bars in Group 2 Element 1 . The variable $M$ is the pixel size of the object which is 780 in this case. The critical distance $z_{c}$ is then calculated to be $33.25 \mathrm{~cm}$, and the critical angle $\theta_{c}$ is $1.33^{\circ}$. Although this critical distance is satisfactory to resolve any element in the USAF chart by itself, the virtual image of the complete chart will be insufficiently spatially separated from the zero-order term upon reconstruction. The true critical distance is then larger and can be found by taking the size of the chart to be approximately $D=4.4 \mathrm{~mm}$ which yields a distance of $z_{c}=60.66 \mathrm{~cm}$. With the aforementioned considerations, a distance of $z=100 \mathrm{~cm}$ and angle of $\theta=0.03$ were selected. The off-axis angle was the optimal value identified by Minniti [4].

Now having an ideal test hologram to work with the next step was to create a more realistic hologram that contains the noise generated after having passed through an optically scattering environment. The scattering comes from a collection of equally-sized spherical water droplets, with a refraction index $n$ of 1.33 , and a number density $\rho_{N}$ of $10^{6} \mathrm{~cm}^{-3}$. This configuration will be referred to as Study 1 , and consists of three cases where the optical depth OD is gradually increased while the particle radius is kept constant at $5 \mu \mathrm{m}$. The first case has a nominal OD of 1 , the next has $O D=3$, and the final case is with $O D=7$. SimuHolo provides the perfect hologram as a BMP file, which is then inserted into the Zemax environment using the "Slide" object. A collimated light source behind it illuminates the hologram and its contents which can then be captured on a detector. The key parameters that allow this are found under the "Coat/Scatter" tab in the "Properties" of the "Slide" object. There are several different scatter models available, but the most suitable for illuminating an inserted image file is the "Lambertian" model. The "Scatter fraction" should be set to 1; this parameter determines the percent of rays that scatter off the "Slide" and a value of 1 ensures all of them do.

As outlined before, the "Rectangular Volume" was used to simulate a scattering cell as shown in Fig. 3 below, and an increase in OD was achieved by increasing the length of the scattering cell (while keeping scatterer size and number density fixed). The physical implication of this is that more rays are scattered out of the line of sight which means fewer ballistic rays make it through to the detector, thereby decreasing the unperturbed transmitted intensity.

The laser beam was modeled in OpticStudio with a 400nm wavelength, and the coherence length $L$ was set at $30 \mu \mathrm{m}$. The user has the choice to set the number of rays launched by the ray trace program; one billion rays was found sufficient for all simulations in this paper.

Arriving at a desired value for $O D$ requires a measurement of both incident and unperturbed transmitted intensities. The measurement of the incident intensity is trivially accomplished in OpticStudio by placing a "Detector Rectangle" object in front of the illuminated

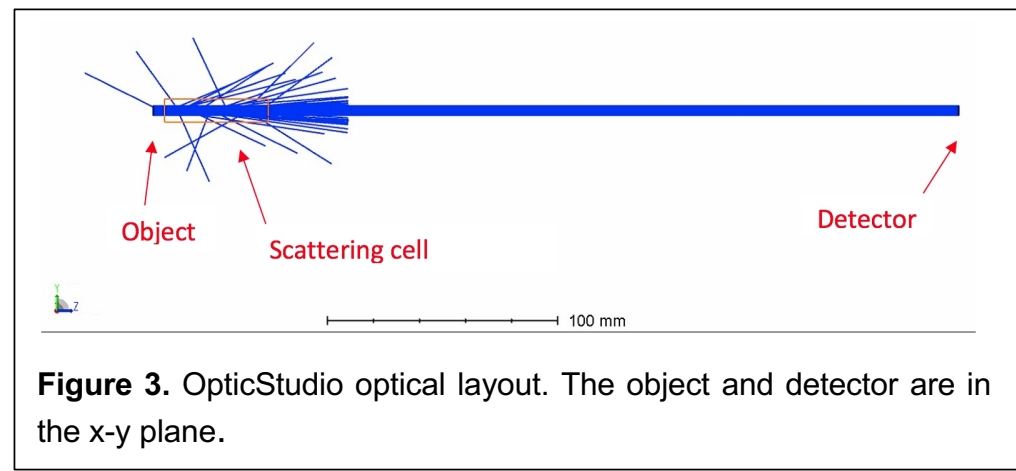


hologram. For simplicity this detector is made the same size as the hologram. The measure of the transmitted intensity needs be approached more carefully due to the definition of it being the intensity from ballistic rays only. Placing the detector in front of the scattering cell results in an artificially high transmitted intensity because it includes the contributions of mildly scattered rays hitting the detector. This problem is solved by placing the detector far from the scattering source so that only ballistic rays hit the detector. In a laboratory setting, there is a limit to this source-to-detector spacing but not in the simulation, and hence we use a 1 kilometer distance to provide an ideal measure of OD.

For frequency domain cropping, in OD 1 and 3 cases the square cut out was 400 pixels wide which contained enough sampling data, but also did not suffer from excessive noise crosstalk with the DC term. However, with this cut out for $\mathrm{OD}=7$ the reconstruction was hidden behind too much noise to resolve any elements beyond Group 3, and so a 200-pixel wide cut out was found to be the balance between sampling data, the more of which improves resolution, and noise. The holograms, and their corresponding reconstructions are shown in Fig. 4. The smallest resolvable element for OD of 1 is Group 5 element 3, which has a size of $12.40 \mu \mathrm{m}$. For OD 3 it is Group 5 element 2, which corresponds to $13.92 \mu \mathrm{m}$. The harshest scattering environment produced here has an OD value of 7 , and in this case it is possible to resolve up to Group 4 element 4, which corresponds to $22.10 \mu \mathrm{m}$. Note that the OD 7 reconstruction lost significant sampling data in order to reduce noise levels, and in the process produced distorted elements in Groups 4 and 5 which were previously resolvable.

The optical depth parameter on its own is not sufficient to describe a scattering environment, and we show in Study 2 that by varying the particle radius at a constant OD the noise levels and therefore resolution changes. Most of the controlled variables from Study 1 were kept with the exceptions of the particle number density and radius. The number density is varied because as particle radius increases the number density should decrease in order to maintain a fixed OD value of 7 . For Study 2 , the scattering cell length is fixed at $45 \mathrm{~mm}$. This study looks at three cases of varying scatterer radius: $1 \mu \mathrm{m}, 5 \mu \mathrm{m}$ (already done in Study 1 ), and $15 \mu \mathrm{m}$. The number density corresponding to each radius that produced OD $=7$ was found by changing the number density until the transmitted intensity matched the value predicted by the optical depth equation.

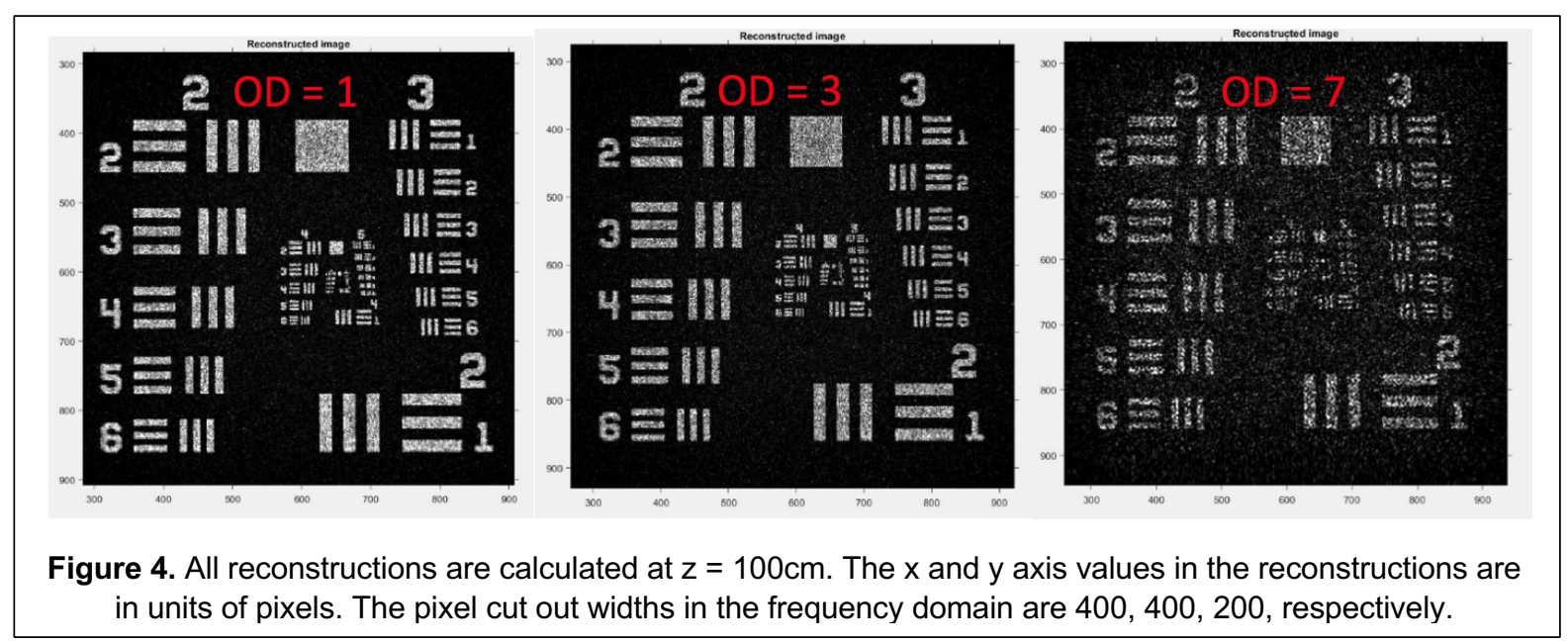


For consistency, the pixel width cut out in the frequency domain was fixed at 200 pixels for all three cases in Study 2. As expected, because of the difference in scattering efficiency from different sized particles, and although they are all characterized by the same OD, the number of rays hitting the detector varies significantly. As the particle radius increases so does the number of scattered rays. The number of ballistic rays has an upper limit set for a constant OD which for our ray traces with a billion launched rays gives about 800,000 ballistic rays reaching the detector, so any excess rays measured in a simulation have to be scattering. For case 1 with a particle radius of $1 \mu \mathrm{m}$ the total number of rays hitting the detector is 900,000 , and for radius $5 \mu \mathrm{m}$ it is $1,700,000$ rays, and for radius $15 \mu \mathrm{m}$ it is $5,600,000$ rays. The difference between the total number of rays, and the maximum number of ballistic rays is the number of scattering rays that perturb the image, which leads to different qualities in reconstruction, as shown in Fig. 5. The harshest scattering environment has the largest particle radius of $15 \mu \mathrm{m}$,

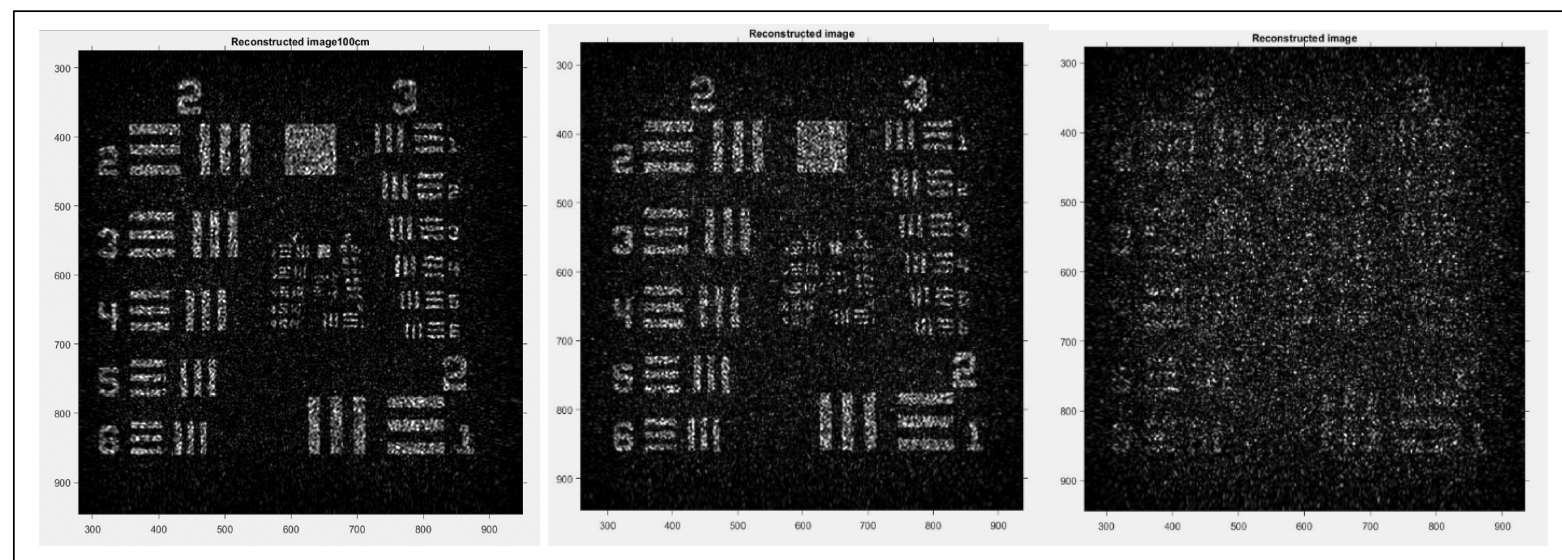

Figure 5. Study 2 reconstructions at $z=100 \mathrm{~cm}$ and a constant OD of 7 . The $x$ and $y$ axis are in units of pixels. The pixel cut out widths in the frequency domain are a constant 200.

and allows only the elements from Group 2 to be resolved, corresponding to a resolution of $70.15 \mu \mathrm{m}$. In the radius $=1 \mu \mathrm{m}$ reconstruction the smallest resolvable element is 3 in group 4 , corresponding to $24.80 \mu \mathrm{m}$, and the same goes for the $5 \mu \mathrm{m}$ radius case although the higher noise levels produce a weak contrast with the black background.

\section{Conclusions}

This paper introduces a new method of simulating off-axis digital holography imaging systems. Preliminary results show the use of a "perfectly" simulated USAF resolution chart hologram produced with a MATLAB script being taken through a Zemax environment and subjected to scattering noise and optical aberrations. The reconstructions agree with expected outcomes previously suggested in experimental measurements that a scatterer should be parametrized by more than one variable, and that a lensed configuration improves image quality.

The size of the scatterer plays a central role in the lower limits of resolution as it was shown that for a given value of optical depth, the particles themselves become recorded in the hologram, and are part of the reconstructed image. Hence for fixed OD, the more light reaching the detector the more of the scattering particle objects interfere with the target image. In laboratory conditions (as for example in a diesel spray environment), the particle scatterers are not a singular size but are rather a distribution of particle sizes. In future work, a hyperrealistic scatterer could be simulated in the Zemax OpticStudio workspace that reflects a realworld distribution. 
The limitation of using a "perfect" hologram is identified as being due to the fact that the MATLAB script used, SimuHolo, or any code in fact, takes in a finite-pixeled image file as the object, and thus produces a hologram with less pixel data than a real holographic system would produce. A limitation with Zemax is the number of rays launched in the ray trace, and it is recommended that future efforts use the maximum number available in the software which is currently 4 billion. For example, the problem of the visible black spots gaps in the USAF chart bars in the reconstructions can be relieved by increasing the number of rays launched. An experimentally obtained hologram would not suffer from the limitations described here, but rather should yield a sound theoretical basis for which to compare with experimental results.

\section{Acknowledgments}

This material is based upon work supported by, or in part by, the U. S. Army Research Laboratory and the U. S. Army Research Office under contract/grant numbers W911NF1710061 and W911NF2010102.

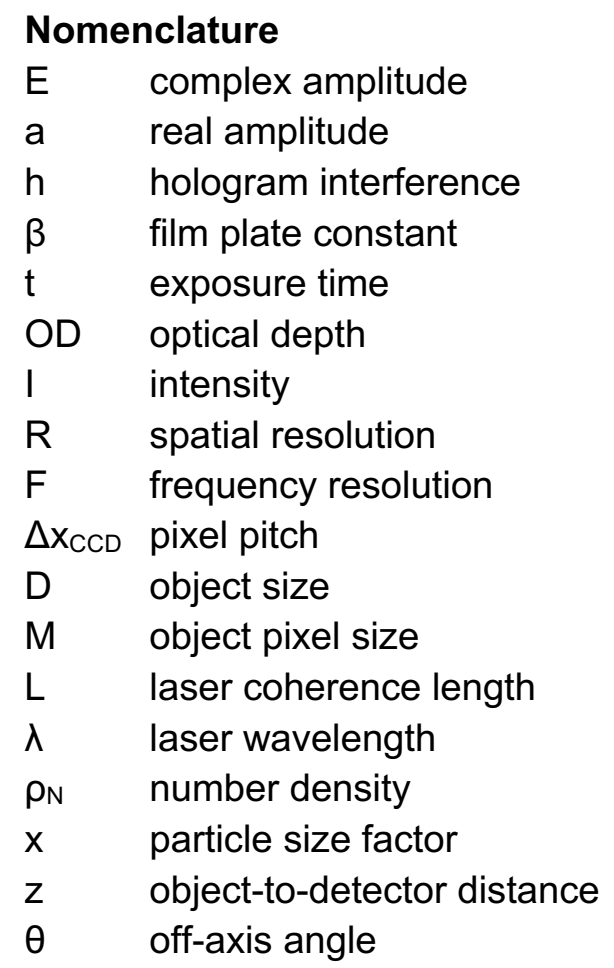

\section{References}

[1] N. Abramson, Light-in-flight recording by holography, Opt. Lett., OL 3 (1978) 121-123.

[2] P. Hariharan, Basics of Holography, Cambridge University Press, 2002.

[3] M. Minniti, Femtosecond Holography of Sprays, UC Irvine, 2019.

[4] M. Minniti, A. Ziaee, J. Trolinger, D. Dunn-Rankin, ULTRASHORT PULSE OFF-AXIS DIGITAL HOLOGRAPHY FOR IMAGING THE CORE STRUCTURE OF TRANSIENT SPRAYS, AAS 28 (2018).

[5] A. Ziaee, C. Dankwart, M. Minniti, J. Trolinger, D. Dunn-Rankin, Ultra-short pulsed off-axis digital holography for imaging dynamic targets in highly scattering conditions, Appl. Opt., AO 56 (2017) 3736-3743.

[6] T.-C. Poon, J.-P. Liu, Introduction to Modern Digital Holography: With Matlab, Cambridge University Press, 2014. 